\title{
Can Subclinical Inflammatory Markers Predict Birth Time and Birth Weight in Hyperemesis Gravidarum?: A Comparative Study and Comprehensive Current Literature Review
}

\author{
๑ Melike Demir Caltekin, ๑ Ibrahim Caltekin*, ๑ Taylan Onat, ๑ Demet Aydogan Kirmizi, \\ ๑ Emre Baser, ๑ Serenat Eris Yalcin**, ๑ Mustafa Kara***, ๑ Ethem Serdar Yalvac \\ Yozgat Bozok University Faculty of Medicine, Department of Obstetrics and Gynecology, Yozgat, Turkey \\ *Yozgat Bozok University Faculty of Medicine, Department of Emergency Medicine, Yozgat, Turkey \\ ** University of Health Sciences Turkey, Antalya Training and Research Hospital, Clinic of Obstetrics and Gynecology, Antalya, Turkey \\ ***Kırsehir Ahi Evran University Faculty of Medicine, Department of Obstetrics and Gynecology, KIrsehir, Turkey
}

\section{Abstract}

Aim: To evaluate subclinical inflammatory markers in hyperemesis gravidarum (HEG) cases and to determine the relationship of these markers with the gestational age at delivery and birth weight in pregnant women with HEG.

Methods: Fifty-two patients who presented to our hospital between 1 May 2017 and 1 September 2019 with HEG and 60 pregnant women as the control group were included in this retrospectively designed study. The relationship of subclinical inflammatory markers such as the neutrophil-to-lymphocyte ratio (NLR), the platelet-to-lymphocyte ratio (PLR), the lymphocyte-to-monocyte ratio (LMR) with HEG, and their relationship with birth week and birth weight in HEG cases were examined.

Results: In HEG cases, NLR and PLR values were higher ( $p=0.006$ and $p=0.004$, respectively), whereas LMR values were lower $(p<0.001)$. In HEG cases, gestational age at delivery had a negative correlation with NLR and PLR and positive correlation with LMR ( $r=-$ $0.567, p<0.001 ; r=-0.322, p=0.02$, and $r=0.279 p=0.045$, respectively). In addition, NLR and PLR had negative correlations with birth weight ( $r=-0.582, p<0.001 ; r=-0.302, p=0.029$, respectively).

Conclusion: While NLR and PLR values increase in HEG cases, LMR value decreases. It has been determined that varying rates of subclinical inflammatory markers in HEG are associated with preterm birth week and low birth weight.

Keywords: Hyperemesis gravidarum, subclinical inflammation, birth weight

\section{Introduction}

Hyperemesis gravidarum (HEG) is a clinical condition that leads to severe nausea, vomiting, weight loss, and electrolyte imbalances in pregnancy, and in some cases, hospitalization is required (1). However, there is no consensus with definitive diagnostic criteria that can define this presentation and multifactorial effects have been mentioned in the etiology (2). HEG may be associated with maternal and fetal poor outcomes and it remains to be the leading cause of hospital admissions and hospitalizations in early pregnancy weeks $(3,4)$.
There are no markers in use to diagnose HEG or to determine the severity of the disease (1). However, it has been stated that inflammation plays an effective role in HEG, and many inflammatory markers play a role in the determination of this process (5). Subclinical inflammatory markers such as the neutrophil-to-lymphocyte ratio $(N L R)$, the platelet-to-lymphocyte ratio (PLR), and the lymphocyte-to-monocyte ratio (LMR) are used in other inflammatory diseases in the determination of the disease activity and severity and in making the diagnosis (6-8). In addition, C-reactive protein (CRP) levels may increase in

Yazışma Adresi/Address for Correspondence: Melike Demir Caltekin, Yozgat Bozok University

Faculty of Medicine, Department of Obstetrics and Gynecology, Yozgat, Turkey

Phone: +90 3542127050 E-mail: melike_deu@hotmail.com ORCID: orcid.org/0000-0001-8797-7794

Received: 11.12.2020 Accepted: 30.01.2021

'Copyright 2021 by The Medical Bulletin of istanbul Haseki Training and Research Hospital The Medical Bulletin of Haseki published by Galenos Yayınevi. 
HEG, where inflammatory mechanisms play a critical role in pathophysiology (9). Just like CRP, NLR and PLR, which are important subclinical inflammatory markers, have also been found to be increased in HEG (10). Also, systemic inflammatory markers are independent determinative markers for systemic endothelial dysfunction (11). It has been determined in different pregnant groups that these markers can be predictors for both low neonatal birth weight and preterm birth week (12).

In this study, it was aimed to evaluate subclinical inflammatory markers in pregnant women with HEG and to determine the relationship of these markers with the birth week and birth weight.

\section{Methods}

The ethics committee approval of the study was given by the university's local ethics committee (protocol number: 2020-KAEK-189_2020.05.19_20) and the principles of the Helsinki Declaration were followed during the study. The study was conducted in a tertiary center and the data of the patients were obtained from the electronic medical records of the hospital. Fifty-two patients who presented to our hospital with HEG between May $1^{\text {st }} 2017$ and September $1^{\text {st }} 2019$ and 60 pregnant women as controls were included in this retrospectively designed study. The patients in the study group and the control group were compared in terms of demographic data, gestational characteristics, and inflammatory markers. A power analysis was conducted using the GPower version 3.1.7 software based on the findings of comparable studies (13). An effect size of 0.73 was used with a power set at 0.95 and alpha at 0.05 to determine that an $n=50$ sample size was required in each group.

The demographical and clinical characteristics of the pregnant women who applied to the clinic with symptoms of HEG such as age, gestational age at the time of admission, gravidity, parity, birth weight, and the weight gained during pregnancy were determined. The height, the weight, clinical status and clinical course, blood samples, the files, and electronic records of pregnant women were examined, subclinical inflammatory markers such as NLR, PLR, and LMR were determined and body mass index (BMI) was calculated from body weight/height ${ }^{2}$ $\left(\mathrm{kg} / \mathrm{m}^{2}\right)$. The blood values of HEG cases were calculated from the results of blood samples taken at the time of the presentation where the most severe symptoms were seen, before hospitalization and hydration. Blood samples taken from healthy pregnant women without HEG in the first trimester were used in the study for control purposes. The relation of subclinical inflammatory markers with HEG and their relationship with preterm birth week and LBW in HEG cases were also investigated.
The pregnant women who were under 18 years of age, who had multiple pregnancies, smoking history, thyroid disease, $\mathrm{BMI}$ of $>35 \mathrm{~kg} / \mathrm{m}^{2}$, gastrointestinal system disease, chronic liver disease, diabetes mellitus, metabolic syndrome, deep vein thrombosis, rheumatic disease, an inflammatory disease that may affect hematological inflammatory parameters were all excluded from our study $(n=6)$. In addition, patients with conditions such as previous preterm birth, premature rupture of the membranes, and pre-eclampsia that may cause LBW and early gestational week were also excluded from the study $(n=4)$. Patients who had missing data and whose blood sample results were inaccessible were also not included in the study $(n=6)$. A total of 16 pregnant women with HEG were excluded. Considering these features, pregnant women who did not have any chronic or inflammatory disorders and who had the same demographic characteristics as the study group were determined as the control group.

\section{Evaluation of HEG}

A pregnant woman who had more than 2 severe vomiting episodes, the presence of ketonuria in a random urine sample, and weight loss of more than $5 \%$ of body weight was diagnosed with HEG (14).

\section{Laboratory Analysis}

Hematological parameters were studied within the first hour to prevent errors in parameters from blood samples taken in tubes containing Ethylenedinitrile-tetraacetic acid with the $\mathrm{XN}-1000$ hematology analyzer (Sysmex Corporation, Kobe, Japan) device. NLR, PLR, and LMR values were calculated from the obtained complete blood count (CBC) values. NLR was calculated as the absolute neutrophil count divided by the lymphocyte count. PLR was calculated as the absolute platelet count divided by the lymphocyte count. LMR was calculated as the absolute lymphocyte count divided by the monocyte count.

\section{Statistical Analysis}

Statistical Package for Social Sciences (Inc; Chicago, IL, USA) version 20.0 software was used to analyze the data. HEG and control patients' values were determined using visual (histograms, probability plots) and analytical methods (Kolmogorov-Smirnov/Shapiro-Wilk's test) to evaluate whether they were normally distributed or not. The independent sample t-test was used to compare continuous variables with normal distributions and the Mann-Whitney $U$ test was used to compare variables with non-normal distributions. The chi-square test or Fischer's Exact test (when chi-square test assumptions did not hold due to low expected cell counts), where appropriate, was used to compare the proportions in different groups. Analysis results of different variables were given as mean 
\pm standard deviation, median (minimum-maximum), and $\mathrm{n}$ (\%). Receiver operating characteristic (ROC) curve analysis was used to identify the optimal cut-off values of NLR, $P L R$, and LMR in order to diagnose HEG with maximum sensitivity and specificity. Spearman correlation analysis was used for the evaluation of a possible correlation between different variables. A value of $p<0.05$ was accepted as statistically significant.

\section{Results}

The demographic and clinical characteristics of the patient and the control groups are shown in Table 1. HEG cases were found to have earlier birth weeks than the control group $(p<0.001)$. Also, when the weight gained during pregnancy was examined, it was found that this value was lower in HEG cases $(p<0.001)$. It was determined that $61.5 \%$ of the pregnant women who were hospitalized due to hyperemesis presented to the emergency department and $38.5 \%$ presented to the outpatient clinic. The mean hospitalization week of the pregnant women with HEG was 9.6 2 2.4. The average length of hospital stay was determined as $3.1 \pm 2$ days.

The evaluation of subclinical inflammatory markers and hematological values for both groups are shown in Table 2. In HEG cases, NLR and PLR values were higher $(p=0.006, p=0.004$, respectively), whereas $L M R$ values were found to be lower $(p<0.001)$.

\begin{tabular}{|c|c|c|c|c|}
\hline & & $\begin{array}{l}\text { Hyperemesis } \\
(n=52)\end{array}$ & $\begin{array}{l}\text { Control } \\
(n=60)\end{array}$ & p \\
\hline \multicolumn{2}{|l|}{ Age } & $28(19-39)$ & $30(21-37)$ & $0.131^{* *}$ \\
\hline \multicolumn{2}{|l|}{ Gravidity } & $2(1-6)$ & $3(1-6)$ & $0.085^{* *}$ \\
\hline \multicolumn{2}{|l|}{ Parity } & $1(0-4)$ & $1(0-4)$ & $0.080 * *$ \\
\hline \multicolumn{2}{|l|}{$\operatorname{BMI}\left(\mathrm{kg} / \mathrm{m}^{2}\right)^{\wedge}$} & $26.5 \pm 4.36$ & $27.3 \pm 3.41$ & $0.288^{*}$ \\
\hline \multicolumn{2}{|c|}{ Birth weight $(\mathrm{g})^{\wedge}$} & $3060 \pm 321$ & $3265 \pm 414$ & $0.004^{*}$ \\
\hline \multicolumn{2}{|c|}{ Gestational age at delivery } & $38(34-42)$ & $39(35-42)$ & $<0.001^{* *}$ \\
\hline \multicolumn{2}{|c|}{$\begin{array}{l}\text { Weight gained during } \\
\text { pregnancy }\end{array}$} & $11(4-18)$ & $13(7-22)$ & $<0.001^{* *}$ \\
\hline \multirow{2}{*}{ Apgar 1. min } & $<7$ & $3(5.8 \%)$ & $4(6.7 \%)$ & \multirow{2}{*}{$0.845^{* * *}$} \\
\hline & $\geq 7$ & $49(82.7 \%)$ & $56(93.3 \%)$ & \\
\hline \multirow{2}{*}{ Apgar 5. min } & $<7$ & $1(1.9 \%)$ & $2(3.3 \%)$ & \multirow{2}{*}{$0.641 * * *$} \\
\hline & $\geq 7$ & $51(98.1 \%)$ & $58(96.7 \%)$ & \\
\hline \multirow[t]{2}{*}{$\begin{array}{l}\text { Neonatal } \\
\text { gender }\end{array}$} & Male & $21(40.4 \%)$ & $31(51.7 \%)$ & \multirow{2}{*}{$0.315^{\star \star \star}$} \\
\hline & Female & $31(59.6)$ & $29(48.3 \%)$ & \\
\hline \multirow[t]{2}{*}{$\begin{array}{l}\text { Mode of } \\
\text { delivery }\end{array}$} & Vag.delivery & $25(\% 48.1)$ & $24(40 \%)$ & \multirow[t]{2}{*}{$0.504^{* * *}$} \\
\hline & C-section & $27(\% 51.9)$ & $36(60 \%)$ & \\
\hline \multicolumn{5}{|c|}{$\begin{array}{l}\text { A Values are given as mean } \pm \text { standard deviation; others are given as median } \\
\text { (minimum-maximum), }{ }^{*} \text { Independent simple } t \text {-test, }{ }^{* \star} \text { Mann-Whitney } U \text { test, } \\
{ }^{* *} \text { Chi-square test. Values in bold represent statistically significant outcomes. } \\
\text { BMI: Body mass index, Vag.delivery: Vaginal delivery, C-section: Caesarean section }\end{array}$} \\
\hline
\end{tabular}

In Table 3, the correlation analysis of HEG cases with birth week, baby birth weight, $1^{\text {st }}$ and $5^{\text {th }}$ minute Apgar scores and urine ketone level, hospitalization time, and subclinical inflammatory markers are shown. In HEG cases, birth week had negative correlations with NLR and PLR and a positive correlation with LMR $(r=-0.567, p<0.001$; $r=-0.322, p=0.02$, and $r=0.279 p=0.045$, respectively). In addition, NLR and PLR had negative correlations with birth weight $(r=-0.582, p<0.001 ; r=-0.302, p=0.029$, respectively).

ROC analysis of subclinical inflammatory markers for HEG cases is shown in Figure 1. In HEG cases, a 3.65 cut-off value was found with $61.5 \%$ sensitivity and $55 \%$ specificity for NLR, a 113.24 cut-off value was found with $71.2 \%$ sensitivity and $60 \%$ specificity for PLR, and a 3.15 cut-off value was found with $66.7 \%$ sensitivity and $69 \%$ specificity for $L M R$.

\section{Discussion}

In pregnant women who had symptoms of HEG, NLR and PLR values were found to be higher whereas LMR values were lower. Besides, it was determined that the baby birth weight, birth week, and weight gained during pregnancy were lower in HEG cases. In addition, as a result of this study, it was determined that in pregnant women with HEG there was a correlation between subclinical markers and the birth weight and the birth weeks of babies.

\begin{tabular}{|c|c|c|c|}
\hline & $\begin{array}{l}\text { Hyperemesis } \\
(n=52)\end{array}$ & $\begin{array}{l}\text { Control } \\
(n=60)\end{array}$ & p \\
\hline $\mathrm{Hb}(\mathrm{g} / \mathrm{dL})^{\wedge}$ & $12.3 \pm 1.7$ & $12.2 \pm 1.4$ & $0.879^{*}$ \\
\hline $\operatorname{NEU}\left(\times 10^{3} / \mathrm{uL}\right)$ & $\begin{array}{l}7.1 \\
(4.08-12.76) \\
\end{array}$ & \begin{tabular}{|l|}
6.7 \\
$(3.53-12.30)$ \\
\end{tabular} & $0.088^{* *}$ \\
\hline $\operatorname{LYM}\left(\times 10^{3} / \mathrm{uL}\right)$ & $\begin{array}{l}1.7 \\
(0.4-3.2)\end{array}$ & $2.1(1.1-5)$ & $0.015^{* *}$ \\
\hline Monocyte $\left(\times 10^{3} / \mathrm{uL}\right)$ & $\begin{array}{l}0.7 \\
(0.4-1.2)\end{array}$ & $0.5(0.1-1)$ & $<0.001^{* *}$ \\
\hline $\operatorname{PLT}\left(\times 10^{3} / \mathrm{uL}\right)$ & $\begin{array}{l}245 \\
(122-408)\end{array}$ & $\begin{array}{l}214.5 \\
(132-668)\end{array}$ & $0.103 * *$ \\
\hline NLR & $\begin{array}{l}4.1 \\
(2-15.7)\end{array}$ & $\begin{array}{l}3.5 \\
(1.4-7.4) \\
\end{array}$ & $0.006^{* *}$ \\
\hline PLR & $\begin{array}{l}134 \\
(52.7-655.8)\end{array}$ & \begin{tabular}{|l}
105.6 \\
$(49.6-286.7)$ \\
\end{tabular} & $0.004 * *$ \\
\hline LMR & $\begin{array}{l}2.5 \\
(0.4-7) \\
\end{array}$ & $\begin{array}{l}3.7 \\
(1.8-13.4)\end{array}$ & $<0.001$ ** \\
\hline \multicolumn{4}{|c|}{$\begin{array}{l}\text { Nalues are given as mean } \pm \text { standard deviation; others are given as median } \\
\text { (minimum-maximum), *Independent simple t-test, **Mann-Whitney U test. } \\
\text { Values in bold represent statistically significant outcomes. } \\
\text { Hb: Hemoglobin, NLR: Neutrophil-to-lymphocyte ratio, PLR: Platelet-to- } \\
\text { lymphocyte ratio, LMR: Lymphocyte-to-monocyte ratio, NEU: Neutrophil count, } \\
\text { LYM: Lymphocyte count, PLT: Platelet count, HEG: Hyperemesis gravidarum }\end{array}$} \\
\hline
\end{tabular}




\begin{tabular}{|c|c|c|c|c|c|c|c|c|}
\hline & \multicolumn{2}{|c|}{ Gestational age } & \multicolumn{2}{|c|}{ Birth weight } & \multicolumn{2}{|c|}{ APGAR 1. $\min$} & \multicolumn{2}{|c|}{ APGAR 5. $\mathrm{min}$} \\
\hline & $r$ & $p$ & $\mathbf{r}$ & $p$ & $r$ & p & $r$ & $p$ \\
\hline Urine ketone level & -0.173 & 0.22 & -0.057 & 0.688 & -0.095 & 0.504 & -0.102 & 0.470 \\
\hline Length of hospital stay & -0.241 & 0.085 & -0.185 & 0.188 & -0.149 & 0.293 & -0.164 & 0.244 \\
\hline NLR & -0.567 & $<0.001$ & -0.582 & $<0.001$ & 0.239 & 0.088 & 0.190 & 0.177 \\
\hline PLR & -0.322 & 0.02 & -0.302 & 0.029 & 0.201 & 0.153 & 0.257 & 0.066 \\
\hline LMR & 0.279 & 0.045 & 0.253 & 0.071 & -0.073 & 0.606 & 0.014 & 0.922 \\
\hline
\end{tabular}
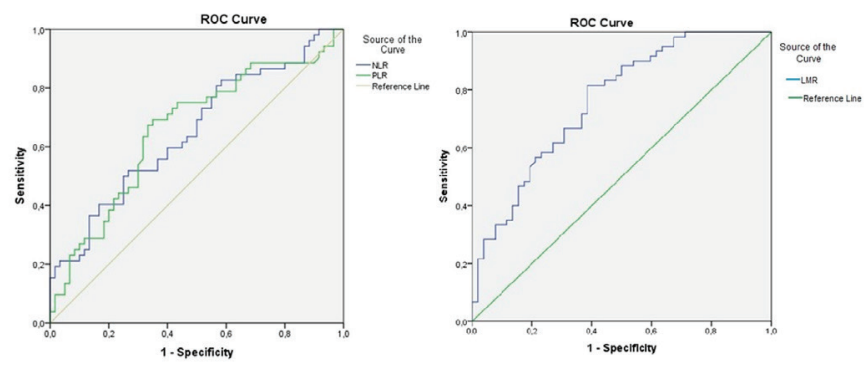

Figure 1. Receiver operating characteristic curves of neutrophilto-lymphocyte ratio, platelet-to-lymphocyte ratio, lymphocyteto-monocyte ratio according to the presence of hyperemesis gravidarum

LMR: Lymphocyte-to-monocyte ratio, PLR: Platelet-to-lymphocyte ratio, NLR: Neutrophil-to-lymphocyte ratio

The etiology of many diseases has been investigated in gynecology and obstetrics practice and it has been determined that inflammatory events play a key role in cases such as polycystic ovarian syndrome, ovarian hyperstimulation syndrome, and pregnancy hypertension $(12,15,16)$. Although there is no test or marker that can fully reveal the mechanisms of HEG formation, the early determinants of inflammation such as interleukin (IL6) and tumor necrosis factor- $\alpha$ were high in HEG cases which indicates an inflammatory condition. Besides, the determinants such as NLR, PLR, and CRP were found to be high in HEG (17), and this has been supported by many studies $(13,18)$. In accordance with these results, in our study, it was determined that there was a decrease in LMR levels, which is another inflammatory indicator, as well as increased NLR and PLR levels in HEG cases.

In our study, the neonatal birth weight of the babies of women with HEG was found to be lower than the control group. Neonatal birth weight is affected by fetal, maternal, and placental factors. In addition, endothelial damage, thrombosis, and inflammation have been shown to constitute the main injury through mechanisms in which inflammatory cells play a role in many diseases. Besides, it has been reported that neonatal birth weight is associated with NLR and PLR (19). In our study, it was determined that there was a negative correlation between increased
NLR and PLR levels, which are inflammatory parameters, and birth weight in HEG cases, and a negative correlation between LMR levels and birth weight.

Low birth weight (LBW) constitutes an increased risk for perinatal mortality and morbidity (20). HEG is known to be associated with neonatal outcomes such as preterm labor, LBW, and placental dysfunctions such as miscarriage and stillbirth $(21,22)$. However, in our study with pregnant women with similar demographic characteristics, it was ensured that LBW in HEG cases could have been predicted with subclinical inflammatory markers.

In HEG cases, the relationship between ketonuria levels and inflammatory parameters have been previously studied, but similar to our study, no significant correlation was found between inflammatory markers (23). Vikanes et al. (24) determined that the $1^{\text {st }}$ minute Apgar scores of $<7$ were less common in HEG cases, and both Kuru et al. (25) and Vikanes et al. (24) have identified the 5th minute Apgar scores of $<7$ as insignificant in cases of HEG.

The correlation of $1^{\text {st }}$ and $5^{\text {th }}$ minute Apgar scores with inflammatory markers was previously investigated in patients with pregnancy hypertension, but no correlation was observed (12). Similarly, in our study, there was no correlation between NLR, PLR, and LMR with $1^{\text {st }}$ and $5^{\text {th }}$ minute Apgar scores in HEG cases. When the 1st and 5 th minute Apgar scores were examined, it was observed that there was no significant difference between pregnant women with HEG and the control group, especially in terms of the score being $<7$. These results indicate that there is no difference between HEG cases and normal pregnant women in terms of fetal well-being.

In a cohort study by Vandraas et al. (26), it was found that the risk of developing preterm delivery below 32 weeks was lower in pregnant women with $\mathrm{HEG}$, but there was a 0.5 day shortening in the gestational period. In another study evaluating pregnant women with HEG and normal pregnant women, it was found that HEG did not change the birth week and delivery type (vaginal delivery, cesarean delivery) (27). In our study, no difference was detected in terms of delivery type, but it was determined that the gestational week of pregnant women with 
HEG was on average 1 week earlier. It was found that inflammatory markers were correlated with this condition. Vogel et al. (28) reported the relationship between the second trimester inflammatory markers with preterm labor. In our study, it can be concluded that the process leading to birth in pregnant women with HEG might be somewhat shorter.

Contrary to our findings, Kuru et al. (25) reported that HEG was not associated with adverse pregnancy outcomes such as LBW and preterm gestation. This difference may be due to the fact that our study was conducted with a relatively small population or that our patients with HEG consisted of patients with more severe symptoms. Perhaps, if we had classified pregnant women with HEG according to their symptoms or included a third group of pregnant women diagnosed with HEG with normal laboratory parameters, we could clarify whether the association of inflammatory parameters with preterm delivery and LBW was due to HEG or variable blood parameters.

Theoretically, increased hemoconcentration due to vomiting can be expected in patients with HEG. Cintesun et al. (23) found that hematocrit and platelet levels did not change in HEG patients (23). These findings were similar in our study. The degree of hemoconcentration can be masked by the physiological decrease in the hematocrit and platelet levels that normally occurs during pregnancy.

There are some limitations to our study. Our main limitation is the low number of patients because our study was conducted as a single-centered study. Also, the fact that our study was designed retrospectively constitutes another limitation. However, in our study, identifying similar demographic and clinical peer groups in both pregnant women with HEG and the control group enabled our evaluations to be healthy.

\section{Conclusion}

In conclusion, high NLR, PLR, and low LMR values in pregnant women with HEG can be a warning for LBW and preterm birth week. In this respect, there is a need for a larger series of studies to be conducted prospectively.

\section{Authorship Contributions}

Concept: M.D.C., Design: M.K., E.S.Y., Data Collection or Processing: I.C., S.E.Y., Analysis or Interpretation: T.O., Literature Search: D.A.K., E.B., Writing: M.D.C.

Conflict of Interest: No conflict of interest was declared by the authors.

Financial Disclosure: The author declared that this study received no financial support.

\section{References}

1. Koot MH, Grooten IJ, van der Post JAM, et al. Determinants of disease course and severity in hyperemesis gravidarum. Eur J Obstet Gynecol Reprod Biol 2020;245:162-7.
2. Nurmi M, Rautava P, Gissler M, Vahlberg T, Polo-Kantola P. Incidence and risk factors of hyperemesis gravidarum: A national register-based study in Finland, 2005-2017. Acta Obstet Gynecol Scand 2020;99:1003-13.

3. Fejzo MS, Trovik J, Grooten IJ, et al. Nausea and vomiting of pregnancy and hyperemesis gravidarum. Nat Rev Dis Primers 2019;5:62.

4. Koot MH, Boelig RC, Van't Hooft J, et al. Variation in hyperemesis gravidarum definition and outcome reporting in randomised clinical trials: a systematic review. BJOG 2018;125:1514-21.

5. Tayfur C, Burcu DC, Gulten O, et al. Association between platelet to lymphocyte ratio, plateletcrit and the presence and severity of hyperemesis gravidarum. J Obstet Gynaecol Res 2017;43:498-504.

6. Jiang Y, Zang M, Li S. Serum PLR and LMR in Behçet's disease: Can they show the disease activity? Medicine (Baltimore) 2017;96:6981.

7. Pan YC, Jia ZF, Cao DH, et al. Preoperative lymphocyte-tomonocyte ratio (LMR) could independently predict overall survival of resectable gastric cancer patients. Medicine (Baltimore) 2018;97:13896.

8. Qin B, Ma N, Tang Q, et al. Neutrophil to lymphocyte ratio (NLR) and platelet to lymphocyte ratio (PLR) were useful markers in assessment of inflammatory response and disease activity in SLE patients. Mod Rheumatol 2016;26:372-6.

9. Engin-Ustun $Y$, Tonguç E, Var T, et al. Vaspin and C-reactive protein levels in hyperemesis gravidarum. Eur Rev Med Pharmacol Sci 2013;17:138-40.

10. Beyazit F, Öztürk FH, Pek E, Ünsal MA. Evaluation of the hematologic system as a marker of subclinical inflammation in hyperemesis gravidarum: a case control study. Ginekol Pol 2017;88:315-9.

11. Arbel Y, Finkelstein A, Halkin A, et al. Neutrophil/lymphocyte ratio is related to the severity of coronary artery disease and clinical outcome in patients undergoing angiography. Atherosclerosis 2012;225:456-60.

12. Okoye HC, Madu AJ, Korubo K, et al. Correlates of neutrophil/ lymphocyte, platelet/lymphocyte, and platelet/neutrophil ratios of neonates of women with hypertensive disease of pregnancy with neonatal birth outcomes. Hypertens Pregnancy 2019;38:105-10.

13. Kurt RK, Güler A, Silfeler DB, Ozçil MD, Karateke A, Hakverdi $\mathrm{AU}$. Relation of inflammatory markers with both presence and severity of hyperemesis gravidarum. Ginekol Pol 2014;85:58993.

14. Erick M, Cox JT, Mogensen KM. ACOG Practice Bulletin 189: Nausea and Vomiting of Pregnancy. Obstet Gynecol 2018;131:935.

15. Çakıroğlu Y, Vural F, Vural B. The inflammatory markers in polycystic ovary syndrome: association with obesity and IVF outcomes. J Endocrinol Invest 2016;39:899-907. 
16. Verit FF, Cetin O, Yildirim O, Keskin S, Yucel O, Yalcinkaya S. Neutrophil to lymphocyte ratio is superior to platelet to lymphocyte ratio as an early predictor of moderate/severe ovarian hyperstimulation syndrome. J Obstet Gynaecol 2014;34:639-43.

17. Kan E, Emektar E, Corbacioglu K, Safak T, Sariaydin T, Cevik Y. Evaluation of relationship between inflammatory markers and hyperemesis gravidarum in patients admitted to emergency department. Am J Emerg Med 2020;38:292-5.

18. Beyazit F, Öztürk FH, Pek E, Ünsal MA. Evaluation of the hematologic system as a marker of subclinical inflammation in hyperemesis gravidarum: a case control study. Ginekol Pol 2017;88:315-9.

19. Akgun N, Namli Kalem M, Yuce E, Kalem Z, Aktas H. Correlations of maternal neutrophil to lymphocyte ratio (NLR) and platelet to lymphocyte ratio (PLR) with birth weight. J Matern Fetal Neonatal Med 2017;30:2086-91.

20. Pallotto EK, Kilbride HW. Perinatal outcome and later implications of intrauterine growth restriction. Clin Obstet Gynecol 2006;49:257-69.

21. Koudijs HM, Savitri Al, Browne JL, et al. Hyperemesis gravidarum and placental dysfunction disorders. BMC Pregnancy Childbirth 2016;16:374.

22. Koren G, Ornoy A, Berkovitch M. Hyperemesis gravidarumIs it a cause of abnormal fetal brain development? Reprod Toxicol 2018;79:84-8.
23. Çintesun E, Akar S, Gul A, et al. Subclinical inflammation markers in hyperemesis gravidarum and ketonuria: A casecontrol study. J Lab Physicians 2019;11:149-53.

24. Vikanes $\AA$ V , Støer NC, Magnus P, Grjibovski AM. Hyperemesis gravidarum and pregnancy outcomes in the Norwegian Mother and Child Cohort - a cohort study. BMC Pregnancy Childbirth 2013;13:169.

25. Kuru O, Sen S, Akbayır O, et al. Outcomes of pregnancies complicated by hyperemesis gravidarum. Arch Gynecol Obstet 2012;285:1517-21.

26. Vandraas KF, Vikanes AV, Vangen S, Magnus P, Støer NC, Grjibovski AM. Hyperemesis gravidarum and birth outcomes-a population-based cohort study of 2.2 million births in the Norwegian Birth Registry. BJOG 2013;120:1654-60.

27. Turgut E, Erkayiran U, Karadag B, Korkmaz V. Determination of the Relationship Between Serum Lactate Level and Antenatal Complications and Early Neonatal Outcomes In Hyperemesis Gravidarum Patients. EJMI 2018;2:70-5.

28. Vogel I, Goepfert AR, Thorsen P, et al. Early second-trimester inflammatory markers and short cervical length and the risk of recurrent preterm birth. J Reprod Immunol 2007;75:13340. 